\title{
A Real-Time Evaluation of Energy Management Systems for Smart Hybrid home Microgrids
}

\author{
Mousa Marzband ${ }^{\mathrm{a}, \mathrm{b}}$, Seyedeh Samaneh Ghazimirsaeid ${ }^{c}$, Hasan Uppal ${ }^{\mathrm{a}}$, Terrence Fernando ${ }^{\mathrm{c}}$ \\ ${ }^{a}$ School of Electrical and Electronic Engineering, Faculty of Engineering and Physical Sciences, Electrical Energy and Power Systems Group, The University of \\ Manchester, Ferranti Building, Manchester, M13 9PL, United kingdom \\ ${ }^{b}$ Department of Electrical Engineering, Lahijan Branch, Islamic Azad University, Lahijan, Guilan, Iran \\ ${ }^{c}$ School of the Built Environment, $4^{\text {th }}$ Floor, Maxwell Building Room 712 (THINKlab), University of Salford, Salford, M5 4WT, United kingdom
}

\begin{abstract}
Real-time energy management within the concepts of home Microgrids (H-MG) systems is crucial for H-MG operational reliability and safe functionality, regardless of simultaneously emanated variations in generation and load demand transients. In this paper, an experimental design and validation of a real-time mutli-period artificial bee colony (MABC) topology type central energy management system (CEMS) for H-MGs in islanding mode is proposed to maximize operational efficiency and minimize operational cost of the H-MG with full degree of freedom in automatically adapt the management problem under variations in the generation and storage resources in real-time as well, suitable for different size and types of generation resources and storage devices with plug-and-play structure, is presented. A self-adapting CEMS offers a control box capability of adapting and optimally operating with any H-MGs structure and integrated types of generation and storage technologies, using a two-way communication between each asset, being a unique inherent feature. This CEMS framework utilizes feature like day-ahead scheduling (DAS) integrated with real-time scheduling (RTS) units, and local energy market (LEM) structure based on Single Side Auction (SSA) to regulate the price of energy in real-time. The proposed system operates based on the data parameterization such as: the available power from renewable energy resources, the amount of non-responsive load demand, and the wholesale offers from generation units and time-wise scheduling for a range of integrated generation and demand units. Experimental validation shows the effectiveness of our proposed EMS with minimum cost margins and plugand-play capabilitities for a H-MG in real-time islanding mode that can be envisioned for hybrid multi-functional smart grid supply chain energy systems with a revolutionary architectures. The better performance of the proposed algorithm is shown in comparison with the mixed integer non-linear programming (MINLP) algorithm, and its effectiveness is experimentally validated over a microgrid test bed. The obtained results show convergence speed increase and the remarkable improvement of efficiency and accuracy under different condition.
\end{abstract}

Keywords: Artificial bee colony, building energy efficiency, home Micogrid, real-time energy management system, local electricity market.

\section{Nomenclature}

ABC artificial bee colony

BES battery energy storage

BIO biomass

CAE compressed air energy storage

CCU central controller unit

COE cost of energy

CTU combustion turbines unit

DAC data acquisition

DER distributed energy resources

DGN diesel generator

DSA double side auction

ECS electrochemical system

EGP excess generated power

Email address: mousa.marzband@manchester .ac .uk, Tel. +44(0) 1613064654, Fax. +44(0) 1613064820 .

Corresponding author (Mousa Marzband)
ESS

FCE

FLY

GEO

GEN

GTD

IREC

LEM

MABC

MCF

MTG

NRL

PSS

PAF

PEM

PV

RR

REN

RLD energy storage system

fuel cells

flywheels

geothermal

gas engine

gas turbine devices

institut de recerca en energia de catalunya

local energy market

multi-period ABC

molten carbonate fuel cell

microturbine generator

non-responsive load

pumped storage system

phosphoric acid fuel cell

proton exchange membrane

photovoltaic

renewable resources

reciprocating engine

responsive load demand 


$\begin{array}{ll}\text { RTD } & \text { real time dispatching } \\ \text { RTEMS } & \text { real-time energy management system } \\ \text { SHT } & \text { small hydro-turbines } \\ \text { SME } & \text { superconducting magnetic energy storage } \\ \text { SOF } & \text { solid oxide fuel cell } \\ \text { SSA } & \text { single side auction } \\ \text { STS } & \text { solar thermal system } \\ \text { TCP } & \text { total consumed power } \\ \text { TGP } & \text { total generated power } \\ \text { UP } & \text { undelivered power } \\ \text { WTG } & \text { wind turbine generator }\end{array}$

\section{Introduction}

While smart grids are known as the future of power systems, home Microgrids (H-MGs) are known as the vital technology to deliver the functional blocks of smart grid on a local scale $[1,2]$. Although the idea of H-MGs seems to be similar to the various areas of operation in the traditional power system, they are different in which they have to be fully capable of autonomous operation in islanded mode [3, 4]. In addition, H-MGs could be formed in a small-scale like a commercial building to as large as a town area. Since high level integration and control of renewable energy and storage devices are expected in H-MGs, their safe operation and control (traditionally known as ancillary services in the power system) is an important issue for the future smart power system. For an islanded H-MG, shortage in generation or excess available generation will often happen during a day because of the variation of weather data like solar irradiation and wind speed variations. In addition, generation shortage might occur when some of the micro sources are out of service for scheduled maintenance or unexpected event or sudden load increase. In this regard, a top-level supervisory control and management system is critical for H-MGs to operate the system with minimum cost and emission within a safe condition. Since it is likely to have shortage or excess in power generation anytime during the daily operation of an islanded H-MG, the energy management system (EMS) design should consider this specification. It is also desired for the EMS design to adapt and compensate itself in real-time to any changes in the types and capacity of the generation and storage assets quickly, without any modification in the EMS, in addition, maximizing the operational efficiency (equivalently minimizing the cost of operation), minimizing the emission [5], maximizing the lifetime of assets [6-9], increasing the reliability of inter-operatability $[10,11]$ or a combination of the above for a multi-objective type EMS [12-15]. The proposed supervisory controllers for the safe and optimal H-MGs operation are categorized as: central energy management system (CEMS) and distributed energy management system (DEMS), where certain advantages and drawbacks have been comparatively reviewed in $[12,16]$. These algorithms are only developed and implemented in the simulation software with specific H-MG structure [8].

in [17], the design of an CEMS is developed in order to obtain the best purchasing price in day-ahead market, as well as to maximize the utilization of existing DER and study the system stability is reported. However, no optimization approach was used in that work. Furthermore, the research work presented in this paper is a continuation of the work by the authors [14], where a framework for considering nondeterministic polynomial-hard (NP-hard) problem with the cheaper version of the software is needed. Moreover, the global optimal solution in the fastest possible time which is an important issue in real-time application development is achieved in comparison with [14]. In this study, a general CEMS framework with a plug-and-play structure is proposed to minimize the operation cost of H-MG. The allows a degree of freedom in the H-MGs operation to automatically adapt the management problem under any changes in the generation and storage resources in real-time to achieve optimal operation, expanding its capabilities from the first-time operation of the H-MG after implementation, to existing real-time H-MG operation. As a result, the proposed CEMS can be considered as a control box, capable of adapting and optimally operating itself with any H-MGs of a given size and integrated types of generation and storage devices by minimizing the cost of operation. For real-time applications, an extensive database of all available generation and storage technologies for H-MG operation (to the best of authors' knowledge) are considered with their mathematical cost functions and operational constraints. Assuming a two-way communication between each asset in the H-MG with the CEMS (inherent feature of H-MG management system), each device can inform the CEMS with its type and capacity at the beginning of connection, and concurrent changes. Furthermore, the proposed CEMS framework consists of a CCU processor unit which includes day-ahead scheduling (DAS) integrated with real-time scheduling (RTS) units, and LEM units based on Single Side Auction (SSA) to clear the price of energy in real-time. The methodology is structured as follows:

1. a real-time flexible CEMS for all types of H-MG;

2. a plug-and-play operational demonstration in real-time and comprehensive mathematical modeling of different generation and storage assets in NRL and Responsive Load Demand (RLD) loads;

3. a comprehensive local electricity market matrix for any islanded $\mathrm{H}-\mathrm{MG}$;

4. a general cost function optimization matrix in real-time based on MABC.

\section{The Proposed CEMS}

For optimal operation of H-MGs, regardless of the EMS being central or distributed architecture, H-MG system developer should be able to make optimal decisions in a short amount of time in real-time operation. Primarily EMS should be able to satisfy plug-and-play operation particularly in larger H-MGs with different players, as such to the EMS should adapt itself to real-time changes in the type and capacity of generation and storage assets. In this study, a comprehensive CEMS is proposed to overcome the problems discussed above where the plug-and-play operation is provided 


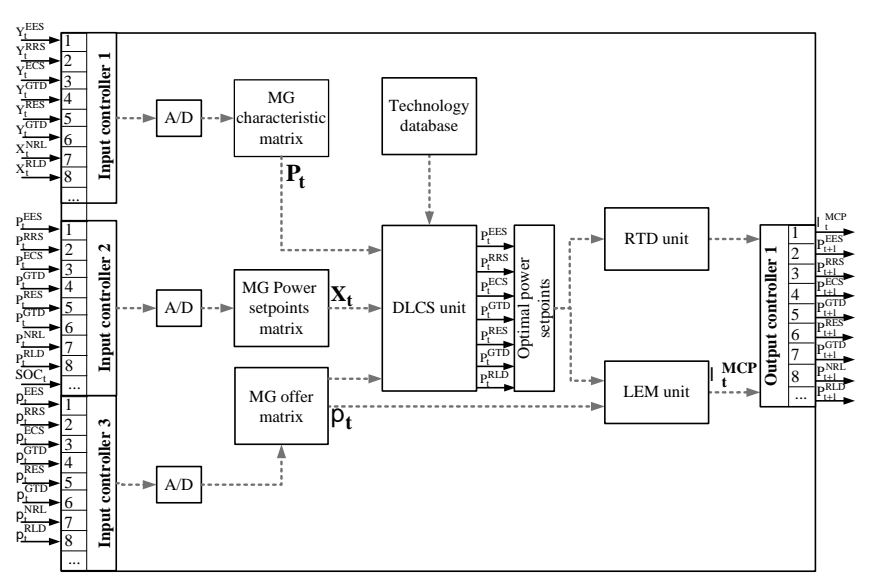

Figure 1: Block-diagram representation of the proposed CEMS

with a database access feature in the CCU (as shown in Figure. 1). The database contains cost function and technical constraints of generation resources, storage devices, and consumers' loads, commonly utilized in H-MGs (to the best of the authors' knowledge), being the core feature of the plugand-play concept where each plugged-in device informs the CCU with its type. The CCU thereby, modifies the overall cost function and technical constraints with the new available technology. The communication link between different devices with the CEMS is also a major feature required for the future smart grids integration, as considered in this study. Although advancements in generation, storage devices, and communications technologies are the primary motivation for $\mathrm{H}-\mathrm{MG}$ implementation, cost optimization of $\mathrm{H}$ MGs with single- and multi-ownership is also critical [14, 17]. In this study, single ownership is considered to simplify the market structure and optimization analysis with features like, e.g., plug-and-play type real-time optimal operation. Nevertheless, the LEM can be implemented for multi-ownership H-MGs.

Another effective feature of the proposed CEMS architecture is that all theoretical considerations for a given MG topology can be easily integrated. For instance, in our case, the main contribution is the experimental validation and implementation of this architecture into a real MG. Various concepts have been considered, such as the digital communication protocols of IEC 61850 application for our MG topology, to emulate each of micro-sources using digital signal processing (DSP), as well as, coordination of transmitted signals from central controller to the DSP and so on, was also achieved. The software packages have been developed in the $\mathrm{C}$ environment to perform each of these actions individually and other tools have also been validated to highlight the effectiveness of the proposed architecture. A detailed description for the proposed EMS-MABC structure is also presented, that extends the advantages of this algorithm topology compared to previous works.

A comprehensive database of all available technologies, different types of DERs and storage devices is categorized into different classes and sub-section unit details are as follows:

\subsection{Technology database}

This is formed with seven different classes of technologies as shown in Figure. 2, that includes all generation resources, storage devices, and consumers' load demand, which are commonly utilized in H-MGs. In each class, similar technologies (only generation and storage) in terms of same type of operational cost and technical constraints recognized for all technologies available in that class are considered. As an illustrative example in Figure. 2, accommodates conventional rotating generation resources with the same operational structure of minimum on and off time, and ramp-up and ramp-down limits. Consumers' loads are also considered in two different categories based on their availability for management: non-responsive load (NRL) and responsive load demand (RLD). The first class includes a part of consumers' critical loads which always must be satisfied regardless of the H-MG situation (e.g., the electricity price, on-peak load hours and so on). The second class, however, contains consumers' responsive loads which are available for demand response (DR) and can participate in the market with their offer price to respond to the utility command to move their power consumption from on-peak to off-peak hours changing the electricity price. HVAC load, Electric Water Heaters (EWHs), and heat pumps are good examples of this category. The price offer of the same class members might be different with others in the same class in the market.

\subsection{H-MG characteristic matrix}

The proposed plug-and play H-MG characteristic matrix structure contains a specific and unique binary (on/off) variable for each class $\left(Y_{t}^{i}, i=1, \cdots, 7\right)$ and individual members of each class ( $X_{t}^{i}, i=$ members of class), as shown in Figure. 3. This approach is similar for all generation resources, storage devices, and consumers' loads available in the technology database and is given as follows:

$$
\begin{aligned}
X_{t}= & \overbrace{\overbrace{t}^{\mathrm{PV}}, X_{t}^{\mathrm{WTG}}, X_{t}^{\mathrm{BIO}}, X_{t}^{\mathrm{STS}}, X_{t}^{\mathrm{GEO}}, X_{t}^{\mathrm{SHT}}, \cdots}^{\overbrace{X_{t}^{\mathrm{BES}}, X_{t}^{\mathrm{BLY}}, X_{t}^{\mathrm{SME}}, X_{t}^{\mathrm{CAE}}, X_{t}^{\mathrm{PSS}}, \ldots}^{Y_{t}^{\mathrm{ELS}}}, \ldots} \\
& \overbrace{X_{t}^{\mathrm{SOF}}, X_{t}^{\mathrm{MCF}}, X_{t}^{\mathrm{PAF}}, X_{t}^{\mathrm{PEM}}, \cdots}^{\overbrace{X_{t}^{\mathrm{DGN}}, X_{t}^{\mathrm{GEN}}, \cdots}^{Y_{t}^{\mathrm{REN}}}, \cdots} \\
& \overbrace{X_{t}^{\mathrm{MTG}}, X_{t}^{\mathrm{CTU}}, \cdots}^{\left.X_{\mathrm{t}}^{\mathrm{RLD}}\right]}
\end{aligned}
$$

As evident from Eq.(1), the proposed plug-and play structure with the H-MG characteristic matrix can be easily expanded to include any new technology type. 


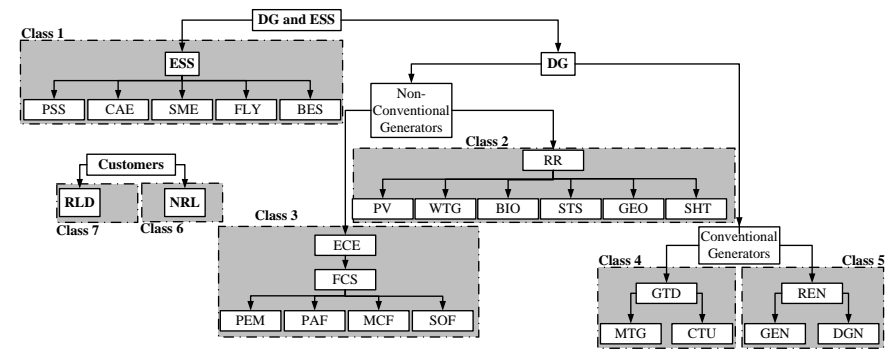

Figure 2: Generation and Storage technology database of the proposed plugand-play structure

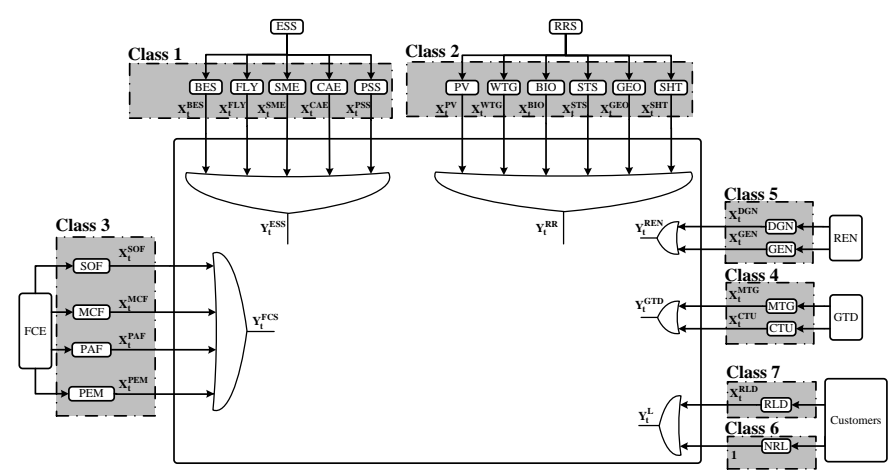

Figure 3: Block-diagram representation of H-MG characteristic matrix

\section{3. $H-M G$ power set-points matrix}

Based on the characteristic matrix in Eq.(1), the scheduled power for each device can be represented by another matrix as follows:

$$
\begin{aligned}
& P_{t}=\overbrace{\left[P_{t}^{P V}, P_{t}^{W T G}, P_{t}^{B I O}, P_{t}^{S T S}, P_{t}^{G E O}, P_{t}^{S H T}, \cdots\right.}^{P_{t}^{R R}} \\
& \overbrace{P_{t}^{B E S}, P_{t}^{F L Y}, P_{t}^{S M E}, P_{t}^{C A E}, P_{t}^{\text {PSS }}, \cdots}^{P_{t}^{E C S S}} \\
& \overbrace{P_{t}^{S O F}, P_{t}^{M C F}, P_{t}^{P A F}, P_{t}^{P E M}, \cdots}^{P_{t}^{\text {REN }}} \\
& \mathrm{P}_{\mathrm{t}}^{\mathrm{REN}} \\
& \overbrace{P_{t}^{D G N}, P_{t}^{G E N}, \cdots}^{P,} \\
& \overbrace{P_{t}^{\text {MTG }}, P_{t}^{C T U}, \ldots}^{P_{t}^{G T D}} \\
& \overbrace{P_{t}^{N R L}, P_{t}^{R L D}}^{P_{t}^{L}}]
\end{aligned}
$$

\subsection{H-MG offer matrix}

In order to develop overall objective function based on operation cost, it is essential to get the offer price from different technologies available in the H-MG. Therefore, a H-MG offer (bid) matrix is defined, where each technology can have its own offer price. The offers from different generation, storage assets and DR is received by the CEMS unit at each time interval, which is then integrated in the same pre-defined H-MG characteristic matrix as follows:

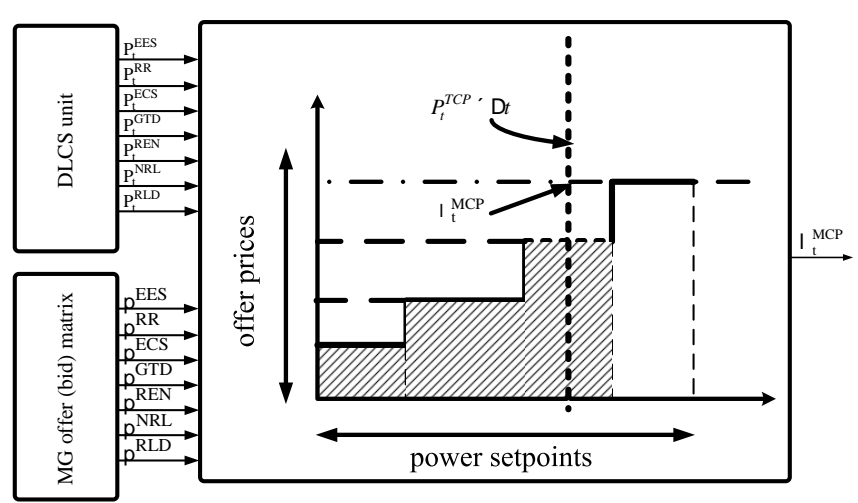

Figure 4: The LEM unit block-diagram representation

$$
\begin{aligned}
& \pi_{\mathrm{t}}=\overbrace{\left[\pi_{\mathrm{t}}^{\mathrm{PV}}, \pi_{\mathrm{t}}^{\mathrm{WTG}}, \pi_{\mathrm{t}}^{\mathrm{BIO}}, \pi_{\mathrm{t}}^{\mathrm{STS}}, \pi_{\mathrm{t}}^{\mathrm{GEO}}, \pi_{\mathrm{t}}^{\mathrm{SHT}}, \cdots\right.}^{\pi_{\mathrm{t}}^{\mathrm{RR}}} \\
& \overbrace{\pi_{\mathrm{t}}^{\mathrm{BES}-}, \pi_{\mathrm{t}}^{\mathrm{FLY}-}, \pi_{\mathrm{t}}^{\mathrm{SME}-}, \pi_{\mathrm{t}}^{\mathrm{CAE}-}, \pi_{\mathrm{t}}^{\mathrm{PSS}-}, \ldots}^{\pi_{\mathrm{t}}^{\mathrm{ESS}-}} \\
& \overbrace{\pi_{\mathrm{t}}^{\mathrm{SOF}}, \pi_{\mathrm{t}}^{\mathrm{MCF}}, \pi_{\mathrm{t}}^{\mathrm{PAF}}, \pi_{\mathrm{t}}^{\mathrm{PEM}}, \cdots}^{\pi_{\mathrm{t}}} \\
& \overbrace{\pi_{\mathrm{t}}^{\mathrm{DGN}}, \pi_{\mathrm{t}}^{\mathrm{GEN}}, \cdots}^{\pi_{\pi_{\mathrm{t}}^{\mathrm{GTN}}}^{\mathrm{REN}}} \\
& \overbrace{\pi_{\mathrm{t}}^{\mathrm{MTG}}, \pi_{\mathrm{t}}^{\mathrm{CTU}}, \cdots}^{\pi_{\mathrm{t}}^{\mathrm{GTD}}}
\end{aligned}
$$

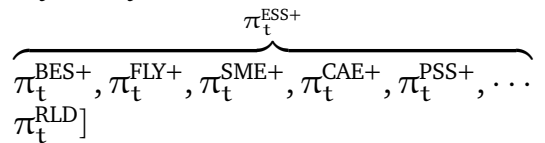

\subsection{LEM unit}

In this section, a LEM is presented to calculate the cost of energy (COE) for the consumers, as represented in Figure. 4. Once the optimization unit performs at each time interval, the LEM unit calculates the market clearing price (MCP) for the consumers, that might be used to calculate the consumers' electricity cost. It is also utilized in this study to show the effectiveness of the proposed CEMS to reduce the MCP. The LEM structure could be formed as single- or doublesided auction model $[14,17]$, SSA and DSA, respectively. A SSA model is a mechanism in which every player only can be buyer or seller, not both simultaneously, and the auction goes to the lowest bidders to cover the electricity demand. In this paper, the SSA-based proposed LEM structure is formed for simple interpretation. The key input features for LEM unit are robust modification to adapt to any market structure without changing the CEMS with optimal setpoints for H-MG offer matrix.

\subsection{RTD Unit}

This unit is responsible for transmitting optimal operation setpoints data from the CEMS unit to the H-MG testbed unit, where transmission speed and data package lost are parameters of optimization $[14,17]$. For optimization by each spe- 


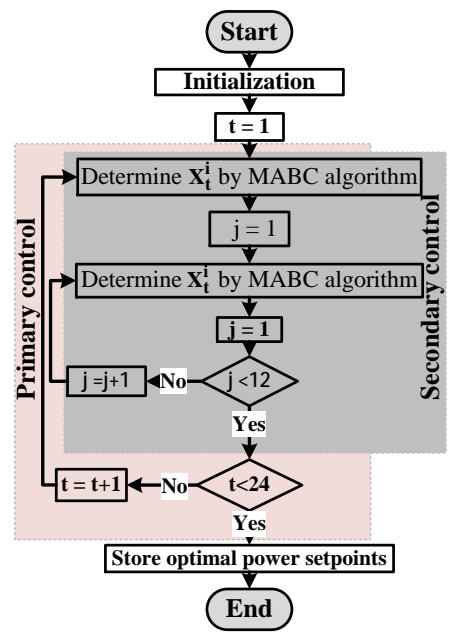

Figure 5: Block-diagram runtime representation of the proposed MABC optimization structure

cific device (emulator), confidential signals are designed between the RTD unit and each individual device in the H-MG testbed. The details on dispatch power set-points from CCU to emulators is discussed elsewhere [17].

\subsection{Double layer control scheme (DLCS) unit}

Conventionally, the $\mathrm{ABC}$ algorithm is also similar to the algorithms based on the innovative methods of a recursive process that starts with an initial population that includes acceptable responses that fulfill consumers requirements). Each input source shows a possible response for solving the problem. So, the initial population of the responses is made up of $\mathrm{N}_{\mathrm{p}}$ number of random D-dimensional vectors, with real values in which each response is defined as the vector $X_{t}^{i}=\left\{X_{t}^{i, 1}, X_{t}^{i, 2}, \cdots, X_{t}^{i, D}\right\}$. This vector determines the position of the $i^{\text {th }}$ input source in the generated population. The parameters that must be given initial value in the MABC optimization technique for each response are the $\mathrm{D}$ number of the variables of the problem, the number of initial food sources $\left(N_{p}\right)$, the maximum number of cycles in which the optimum finding algorithm must be repeated and the limit that shows the maximum number of times for which a response is investigated. If the response is not optimized during a repetition limit, it is considered as left response.

A double layer control scheme (DLCS) including primary and secondary control based on MABC algorithm is implemented to ensure optimum planning to hourly, and real-time operations as shown in Figure. 5 and illustrated by a Pseudocode in Algorithm 1. The control levels as follows:

\subsubsection{Primary control}

A general optimization structure is devised to determine the H-MG technologies set-points at each time interval with minimum cost of operation. The optimization unit includes a general cost-based objective function which is updated in real-time by the H-MG characteristic matrix. Similar to the technology database which contains all available technologies in the H-MG, the optimization unit is required to have

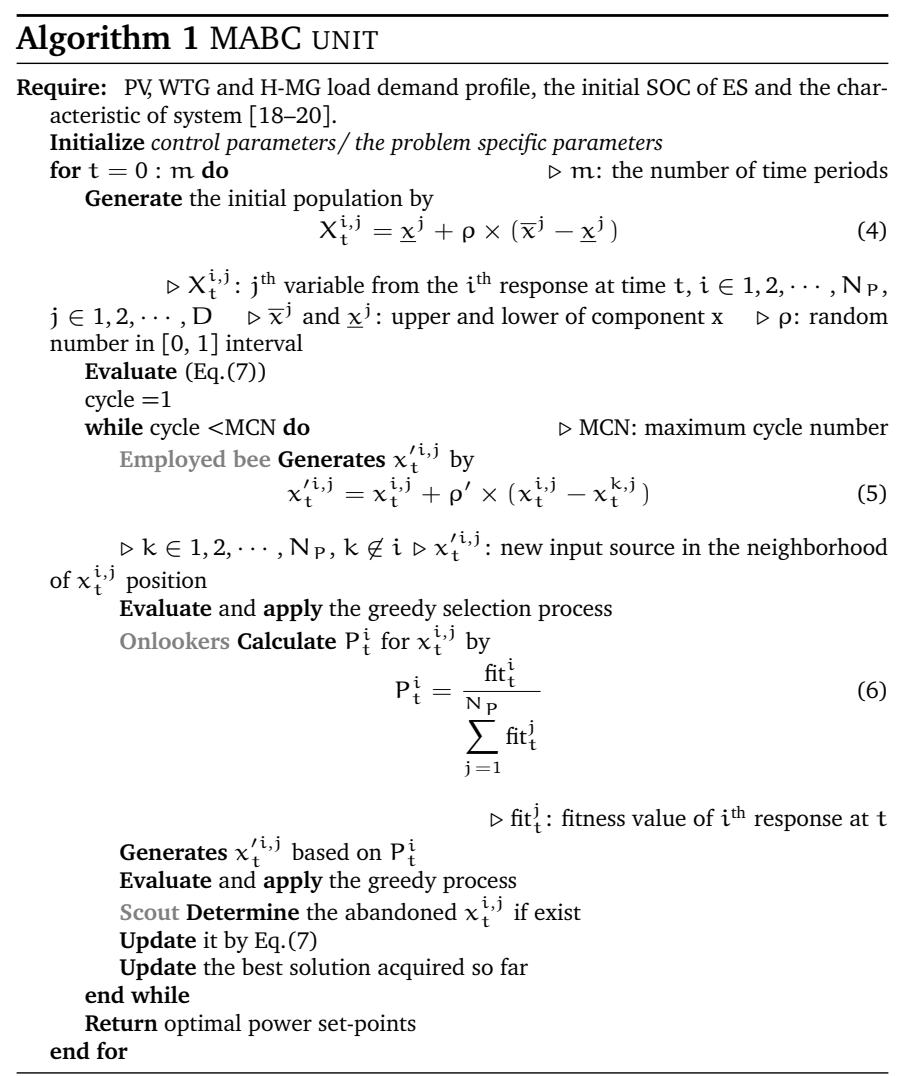

cost function and technical constraints of all available technologies too. As mentioned in Section (2.1), technologies in the same class have similar operational constraints; so seven set of operational constraints can be recognized as a part of the technology database, as an example case.

The ESS class participates with two different offers: charging offer $\left(\pi_{t}^{\mathrm{ESS}-}\right)$ and discharging offer $\left(\pi_{t}^{\mathrm{ESS}+}\right)$. In this study, the H-MG offer matrix is constant throughout a day of operation. The overall objective function of the H-MG optimization problem is defined as follows:

$$
\min \left[\sum_{\forall \mathbf{t}}\left(\begin{array}{c}
\sum_{i=1}^{5} \sum_{j=1}^{n_{i}} X_{t}^{i, j} \times P_{t}^{i, j} \times \pi_{t}^{i, j} \\
-\sum_{i=6}^{7} \sum_{j=1}^{n_{i}} X_{t}^{i, j} \times P_{t}^{i, j} \times \pi_{t}^{i, j} \\
+P_{t}^{U P} \times \pi_{t}^{U P}
\end{array}\right) \times \Delta t\right]
$$

As mentioned earlier, the overall objective function is dynamic, since the H-MG characteristic matrix will be updated in real-time. Similar to the H-MG offer matrix, different offers for charging and discharging are considered for the ESS class. $P_{t}^{U P}$ and $\pi_{t}^{U P}$ are the amount of undelivered power (the unsatisfied part of NRL) at time t and its cost, respectively, as inclusion of this in the objective function serves as a penalty cost for the H-MG operator to avoid undelivered power to the NRL. Objective function is also minimized at each interval (every 5 minutes) for a day to determine the optimal operation setpoints of the H-MG characteristic matrix, which is fixed and used for the rest of the day. This minimization is done for the current time interval and the rest of the day, not 
the previous intervals. In addition, it is this variable generation and load demand forecast is always used in the objective function for the rest of the day, uniquely.

In (7), linear cost function is considered for each class because the optimization unit receives offer from each technology, as its owner is responsible to calculate the cost of electricity in that unit, where the cost function might be linear or nonlinear; however, the H-MG operator's decision is based on the load demand, available power, and their offers, irrespectively.

To satisfy the H-MG and technologies safe operation, several constraints should be included in the optimization unit. Generation and demand balance is a key operational constraint in a power system, which is given as:

$$
\sum_{i=1}^{5} \sum_{j=1}^{n_{i}} X_{t}^{i, j} \times P_{t}^{i, j}=P_{t}^{N R L}-P_{t}^{U P}
$$

In (8), available power from generation resources and storage devices in discharging mode are given in the right-hand side, while load demand (NRL and RLD) and storage devices in charging mode are on the other side, profound terms of interest. $P_{t}^{E G P}$ might be positive (more load is added to utilize excess available generation) or negative (in power shortage), all based on the initial system condition. Therefore, the excess available generation is stored in the RLD and/or ESS in the charging mode.Different constraints for efficient operation of ESS and restrictive optimization constraints of RLD are defined in already as standards [14].

In (9), the scheduled power from classes 2-5 between the minimum and maximum forecasted power or their rated capacity can be limited.

- Generation limit for the micro-sources in classes 2-5

$$
X_{\mathrm{t}} \times\left(\underline{P}_{\mathrm{t}} \leqslant \mathrm{P}_{\mathrm{t}} \leqslant \overline{\mathrm{P}}_{\mathrm{t}}\right)
$$

The generation upper and lower limits can be updated in real-time similar to the H-MG characteristic matrix for variable generation technologies, based on updated forecasts from each unit. Similarly, Technologies available in the GTD class have extra capacity to regulate operational constraints such as; start-up time, ramp-up and Üdown. These requirements are given in (10)-(13).

- Maximum and minimum operating times in the GTD class

$$
\begin{gathered}
{\left[Y_{t}^{\mathrm{GTD}}-\overline{\mathrm{T}}^{i, \mathrm{GTD}}\right] \cdot\left[\mathrm{I}_{\mathrm{t}-1}^{\mathrm{i}, \mathrm{GTD}}-\mathrm{I}_{\mathrm{t}-1}^{\mathrm{i}, \mathrm{GTD}}\right] \geqslant 0} \\
{\left[-\mathrm{Y}_{\mathrm{t}-1}^{\mathrm{GTD}}-\underline{T}^{i, \mathrm{GTD}}\right] \cdot\left[\mathrm{I}_{\mathrm{t}}^{\mathrm{i}, \mathrm{GTD}}-\mathrm{I}_{\mathrm{t}-1}^{\mathrm{i}, \mathrm{GTD}}\right] \geqslant 0}
\end{gathered}
$$

- Ramp-up and ramp-down limits in the GTD class

$$
\begin{aligned}
& {\left[P_{t}^{i, G T D}-P_{t-1}^{i, G T D}\right] \leqslant \bar{R}^{i, G T D}} \\
& {\left[P_{t-1}^{i, G T D}-P_{t}^{i, G T D}\right] \leqslant \underline{R}^{i, G T D}}
\end{aligned}
$$

where $\bar{T}^{i, G T D}$ and $\underline{T}^{i, G T D}$ are maximum and minimum up and down time of unit $i$ in the GTD class (min), respectively, $\bar{R}^{\text {GTD }}$ and $\underline{R}^{\text {GTD }}$ are ramp up and down of unit $i$ in the GTD class $(\mathrm{kW} / \mathrm{min})$, and $\mathrm{I}_{\mathrm{t}}^{\mathrm{i}, \mathrm{GTD}}$ is the operating status of unit $i$ in the GTD class (i.e., $I_{t}^{i, G T D}=1$ when the unit is on, and $I_{t}^{i, G T D}=0$ when it is in off state). $t$ is the current time interval.

At each time interval, new operation settings can be decided with respect to the changes in the H-MG for the proposed CEMS. The restructured CEMS is able to generate the optimal operation set-points for the H-MG based on all available generation, storage, demand and their associated prices. The components of vector $X_{t}^{i}$ in the primary control are determined for each time interval during daily system operation, considering the technical constraints, followed by processing by the secondary control.

\subsubsection{Secondary control}

In the secondary control, real time optimization is performed by including the hourly optimum values derived from the primary control, load values, maximum output power of non-dispatchable generation resources. The load values and the output power of non-dispatchable resources are generated randomly for real time 5 minutes intervals by using the values of hourly forecasting, and by applying designated distribution functions. Each variable of interest is determined by noting the inputs, objective function and the constraints defined in the primary control, considering real-time operation. In addition to the constraints in the primary control, extra constraints are also proposed in the secondary control as follows:

\section{Improving the condition of charge ES [21]}

SOC at the last 5 minute time interval related to each hour of optimization is always greater than $60 \%$ Of its initial value (i.e. $S C_{F} \geqslant 60 \%$ ).

2. Exchanging power with grid

$$
\sum_{j \in J}\left(P_{j}^{\text {GRID- }}-P_{j}^{\text {GRID+ }}\right) \leqslant|J|\left(\bar{P}_{t}^{\text {GRID- }}\right) \pm 5 \%
$$

\section{Power balance}

Power balance between generation and consumption must always be kept at real-time period, as responsive load demand (RLD) is constant for one hour, as same as the value obtained from primary control.

$$
\begin{aligned}
& P_{j}^{\text {WTG }}+P_{j}^{P V}+P_{j}^{\text {MT }}+P_{j}^{\text {BES- }}+P_{j}^{\text {GRID- }}+P_{j}^{P U}= \\
& P_{j}^{n}+P_{t}^{R L D}+P_{j}^{E S+}+P_{t}^{G R I D+}
\end{aligned}
$$

\section{Objective function}

Objective function calculation is based on minimizing the total generation cost in reall-time, raising the share of generating H-MG power and reducing the power required from the grid and increasing the efficiency of BES. 


$$
\min \sum_{\forall t}\left[\begin{array}{c}
\mathrm{P}_{j}^{\mathrm{WTG}} \times \pi^{\mathrm{WTG}}+\mathrm{P}_{j}^{\mathrm{PV}} \times \pi^{\mathrm{PV}}+\mathrm{P}_{j}^{\mathrm{MT}} \times \pi^{\mathrm{MT}} \\
+\mathrm{P}_{j}^{\mathrm{BES}-} \times \pi^{\mathrm{BES}-}+\mathrm{P}_{j}^{\mathrm{GRID}-} \times \pi^{\mathrm{GRID}-} \\
-\mathrm{P}_{j}^{\mathrm{DR}} \times \pi^{\mathrm{DR}}-\mathrm{P}_{j}^{\mathrm{EWH}} \times \pi^{\mathrm{EWH}}- \\
\mathrm{P}_{j}^{\mathrm{BES}+} \times \pi^{\mathrm{BES}+}-\mathrm{P}_{j}^{\mathrm{GRID}+} \times \pi^{\mathrm{GRID}+} \\
+\mathrm{P}_{j}^{\mathrm{UP}} \times \pi^{\mathrm{UP}} \\
+\mathbb{C}_{t}^{\mathrm{PB}}+\mathbb{C}_{t}^{\mathrm{SOC}}+\mathbb{C}_{t}^{\mathrm{GB}}+\mathbb{C}_{t}
\end{array}\right]
$$

where $\mathbb{C}_{t}^{\mathrm{PB}}$ represent the cost due to unbalance between the generated and consumed power (mismatch power cost), calculated as:

$$
\mathbb{C}_{\mathrm{t}}^{\mathrm{PB}}=\pi^{\mathrm{PB}} \times \mathrm{P}_{\mathrm{t}}^{\mathrm{D}}
$$

where the coefficient $\pi^{\mathrm{PB}}$ is considered a great value for preventing unbalance between generation and consumption. $\mathrm{P}_{\mathrm{t}}^{\mathrm{D}}$ can also be calculated as:

$$
\begin{aligned}
& P_{t}^{D}=\left(P_{j}^{W T G}+P_{j}^{P V}+P_{j}^{\text {MT }}+P_{j}^{\text {BES- }}+P_{j}^{\text {GRID- }}\right) \\
& -\left(P_{t}^{\text {DR }}+P_{t}^{\text {EWH }}+P_{j}^{E S+}+P_{t}^{\text {GRID+ }}\right)
\end{aligned}
$$

where $\mathbb{C}_{t}^{S O C}$ is the cost resulting from not fulfilling the SOC achievement condition by the proposed algorithm calculated as:

$$
\mathbb{C}_{\mathrm{t}}^{\mathrm{SOC}}=\beta \times X_{\mathrm{t}}^{\mathrm{SOC}}
$$

where $\beta$ is a very large penalty coefficient. $X_{t}^{S O C}$ is also a binary variable and can satisfy the following relation:

$$
X_{t}^{\mathrm{SOC}}=\left\{\begin{array}{ll}
0 & \text { if } \mathrm{SOC}_{t} \geqslant 60 \% ; \\
1 & \text { otherwise. }
\end{array}\right\}
$$

where $\mathbb{C}_{t}^{G B}$ shows the cost resulting from not achieving power exchange condition with the grid and Can be calculated from the following relation:

$$
\mathbb{C}_{\mathrm{t}}^{\mathrm{GB}}=\gamma \times \mathrm{X}_{\mathrm{t}}^{\mathrm{GB}}
$$

where $X_{t}^{G B}$ is a binary variable which should be employed by:

$$
X_{t}^{G B}=\left\{\begin{array}{ll}
0 & \text { if } \widehat{P}_{t} \geqslant \widehat{P}_{t}^{\prime} \\
1 & \text { if } \widehat{P}_{t}<\widehat{P}_{t}^{\prime} .
\end{array}\right\}
$$

where $\widehat{P}_{t}$ is the difference between the power purchased and sold in each hour in optimizing real time. $\widehat{P}_{t}^{\prime}$ is also the difference between the purchased power and sold power in each time interval in hourly optimization. $\mathbb{C}_{t}$ also shows the difference between the costs obtained from real-time and hourly optimization, as the proposed algorithm numerically minimizes the value of this variable:

$$
\mathbb{C}_{\mathrm{t}}=\delta \times \mathrm{X}_{\mathrm{t}}
$$

where $\delta$ is a very large penalty coefficient. $X_{t}$ can satisfy the following condition:

$$
X_{t}=\left\{\begin{array}{ll}
0 & \text { if } \operatorname{Cost}^{h} \geqslant \operatorname{Cost}^{r t} ; \\
\operatorname{Cost}^{\mathrm{rt}}-\operatorname{Cost}^{\mathrm{h}} & \text { if } \operatorname{Cost}^{\mathrm{h}}<\operatorname{Cost}^{\mathrm{rt}} .
\end{array}\right\}
$$

where Cost $^{\mathrm{rt}}$ and Cost $^{\mathrm{h}}$ are respectively equal to the costs resulting from exploitation in real-time and hourly time optimizing. The above shows that any appropriate heuristic and deterministic algorithms can be used to solve the optimization problem based on CEMS as long as the optimization algorithm should be fast enough to solve the problem

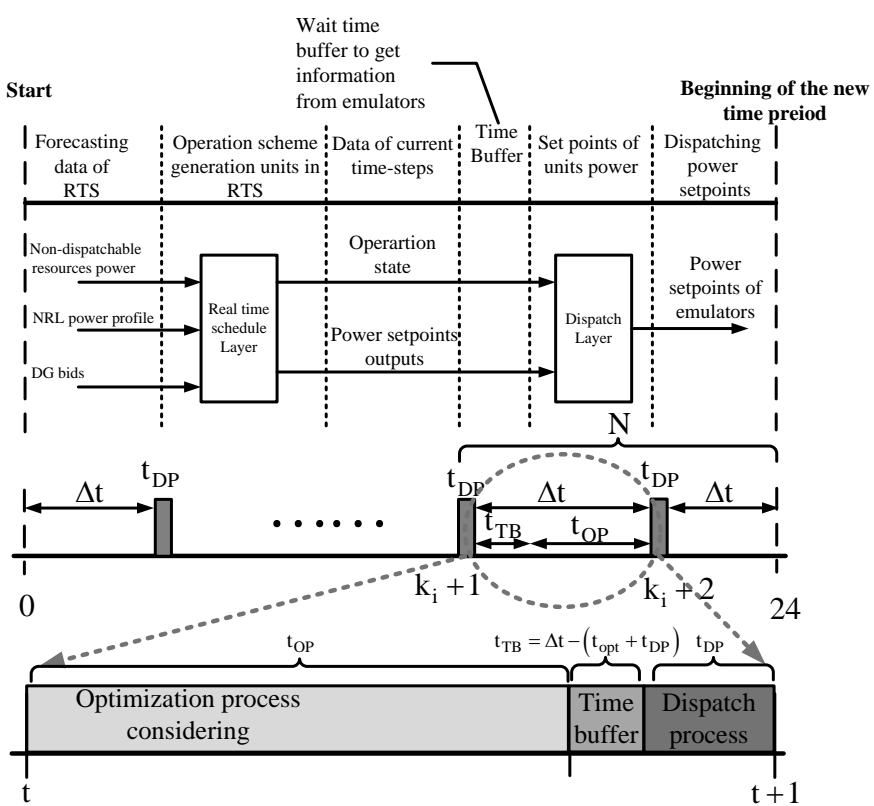

Figure 6: Block-diagram routine representation of the proposed real-time optimization operation for optimal generation and demand forecasts.

with optimal or near optimal solutions, as in our case in less than 5 minutes (including communication delays between the CEMS and individual devices). This feature is facilitated by the multi-period artificial bee colony (MABC) used for DLCS unit to solve the H-MG optimization problem due to the population-based search capability, the simplicity of implementation, adequate convergence speed and robustness [22]. In addition, EMS based on mixed-integer non-linear programming (MINLP) (called hereafter EMS-MINLP) is utilized in the optimization unit [14] and the results obtained are compared with those obtained from EMS-MABC algorithm. MINLP refers to mathematical programming with continuous and discrete variables and non-linearities in the objective function and constraints, similar to the objective function and constraints in this paper. The use of MINLP is a natural approach of formulating problems where it is necessary to simultaneously optimize the system structure (discrete) and parameters (continuous). 


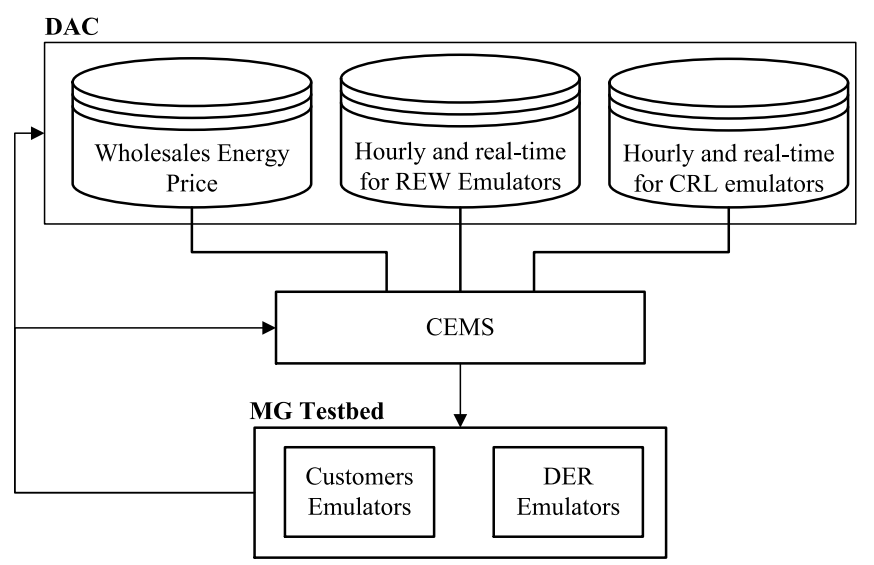

Figure 7: Block-diagram representation of the proposed RTO

Where the proposed optimization approach minimizes cost of real-time operation in the H-MG, it is also desired to guarantee the daily optimum operational routine, not only for the current intervals. Indeed, the proposed MABC algorithm considers the future predictability of information in real-time (i.e., forecasted generation and demand) at each interval for cost minimization. This is advantageous in optimization of time-dependent system since it considers the possible condition of the H-MG in the predicted future (e.g., variation of RES class and the SOC of the ESS). Since the consumers' demand pattern repeats each day, optimization horizon is considered as 24 hours daily cycle. This concept is depicted in Figure. 6. Finally, the optimal set-points will be passed to the LEM unit to calculate the MCP for the consumers, also will be sent to the RTD unit to be dispatched to different devices in the H-MG testbed.

\section{The proposed real time operation (RTO) architecture}

In order to evaluate the performance of the proposed CEMS, real-time emulator environment is adapted with real communication links and delaysas illustrated in proposed RTO of Figure. 7. The method is to model each technology and consumers' load using an emulator in the testbed. The proposed RTO structure includes four main units, namely DAC, CEMS, RTD, and H-MG testbed.

\subsection{DAC Unit}

This unit is responsible for receiving and storing data from different devices in the H-MG, finally it is sent to the CEMS for executing optimal solution of the H-MG operation.

\subsection{CEMS Unit}

This unit contains DLCS unit, technology database, and LEM unit which are explained in details in Section 2.
Table 1: The offers suggested by the micro-sources and the consumers have been presented $[€ / \mathrm{kWh}]$

\begin{tabular}{|c|c|c|c|c|c|c|}
\hline$\pi^{\mathrm{WTG}}$ & $\pi^{\mathrm{PV}}$ & $\pi^{\mathrm{MTG}}$ & $\pi^{\mathrm{BES}-}$ & $\pi^{\mathrm{BES}+}$ & $\pi^{\mathrm{UP}}$ & $\pi^{\mathrm{RLD}}$ \\
\hline 0.083 & 0.1 & 0.15 & 0.145 & 0.125 & 1.5 & 0.105 \\
\hline
\end{tabular}

\subsection{The $H-M G$ Testbed}

The different generation resources and consumers' load with battery storage are considered in the H-MG testbed, as shown in Figure. 8. PV, WTG, and MTG are considered as the generation resources in the H-MG. BES is also considered in the H-MG testbed as storage device. The consumers' load is divided to NRL and RLD. Figure. 9 illustrates the experimental H-MG testbed in the laboratory setup. The designed structure is modular, such as standard experimental practices $[14,17]$ which more emulators for extra generation and storage devices can be added to the system without any further modifications in the running software. Actual real-time operation is performed using WTG, PV, and load demand data $[14,17]$, where the data is sampled every 5 minutes of the same day, where key features are:

1. Sending and receiving data from/to the devices (i.e., emulators) takes $1.5-3$ seconds, with best performance under 3 seconds delay. In addition, the optimization unit spends 3 minutes in average to find the optimal solutions

2. The measured data does not show any meaningful variation in 5 minutes interval

In TABLE 1, the constant offer prices used for different devices are reported.

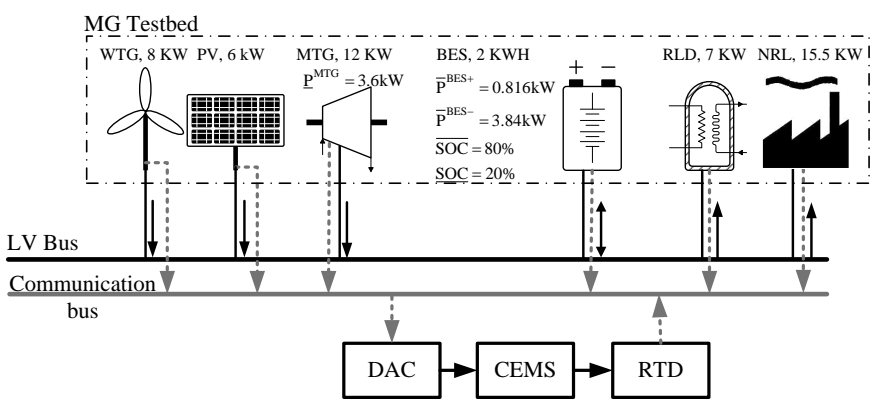

Figure 8: Schematic diagram of the IREC's H-MG testbed

\subsection{The IREC's H-MG System Configuration}

The schematic of the system under study has been shown in Figure. 10.

\section{Results and discussion}

Experimental evaluation over the islanded IREC's H-MG is carried out to verify the EMS operation under different scenarios. The scenarios bellow have been considered for testing the performance and efficiency of the suggested algorithm: 


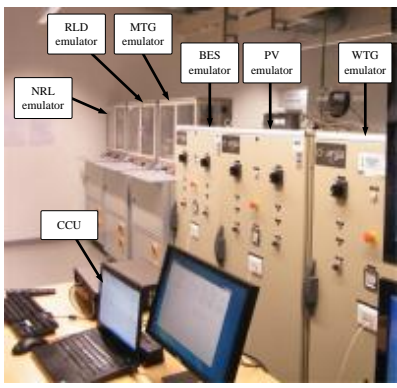

(a) IREC's H-MG

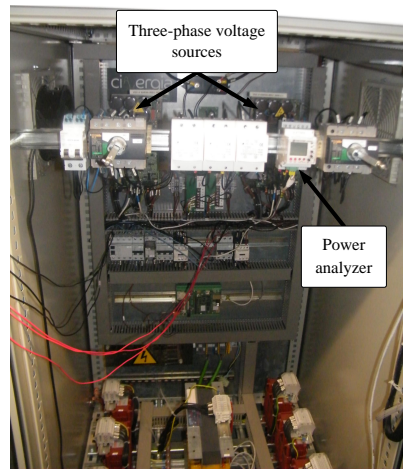

(b) Cabinet inside details
Figure 9: System configuration of IREC's H-MG Testbed

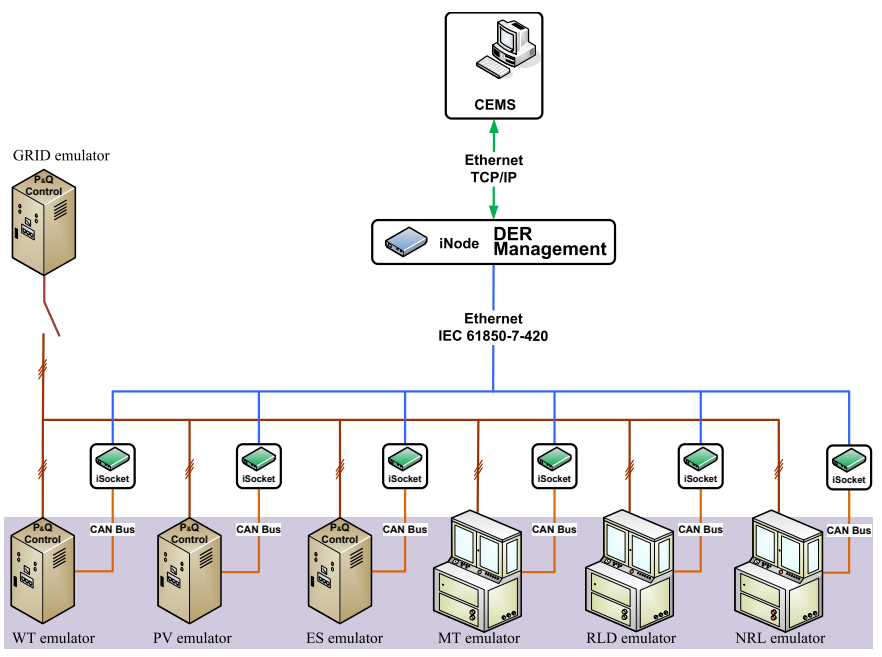

Figure 10: Single line diagram of the system under study

- Scenario $\sharp 1$ : Normal operation (In this scenario, the system is in normal operation mode and the optimum power and the proper timing of each one of the present microsources in the system will be obtained by the suggested algorithm)

- Scenario $\sharp 2$ : Sudden load increase (Sudden load increase is occurred during 17:00-17:30 and 18:00:18:30 periods.)

- Scenario $\sharp 3$ : Plug and play ability (The system plug and play ability can be investigated by this scenario. WTG has shutdown during 19:30 to $21: 00$ periods. Also, PV has shutdown during 19:30-20:00 periods.)

The total consumed power (TCP) by the consumers and the power consumed by each of them are shown in Figure. 11. As it is observed, mainly the RLD load is supplied by proposed algorithm during the periods 00:00-06:00 and 12:00-18:00, where the secondary control tries to fulfil power balance in real-time optimizing performance, where the consumption peak is low and BES has its maximum SOC. During the period 12:00-18:00 after discharging BES and SOC reaching a lower value than the value defined in one of the constraints, the proposed algorithm in the secondary control charges BES,

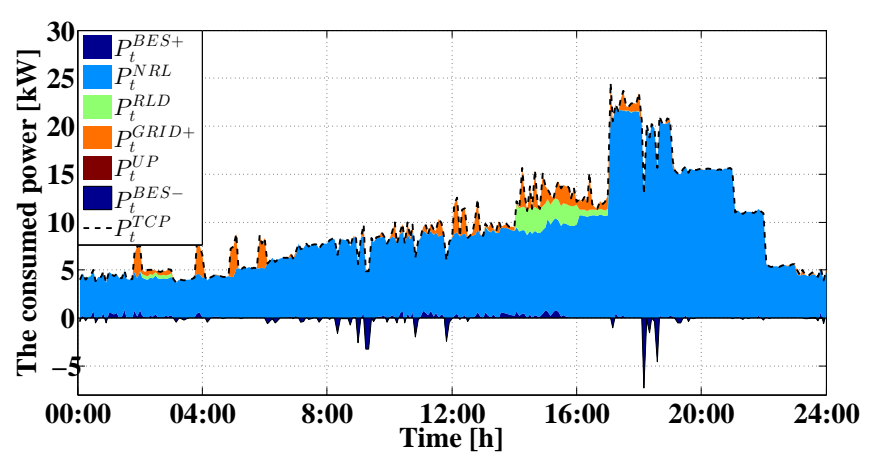

Figure 11: TCP and consumed power by the consumers

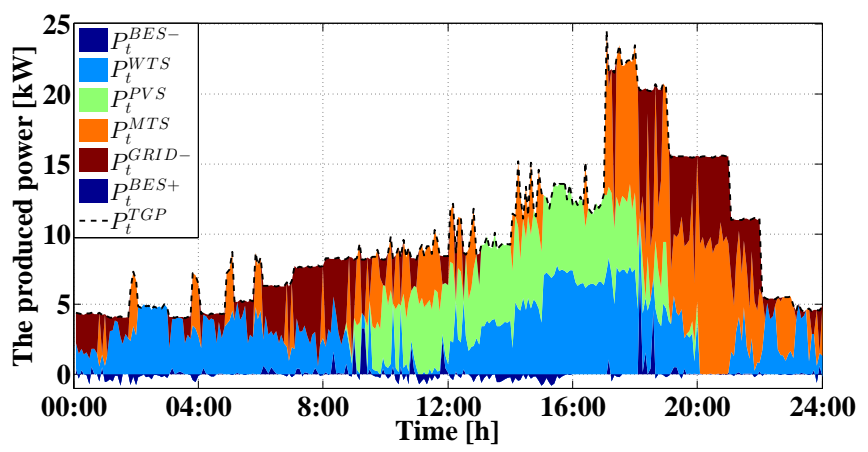

Figure 12: TGP and power generated by the generation resources

such that it fulfils the defined constraints. For daily operation the proposed algorithm frequently tries to sell the excess generated power to the grid. The process circumvents and adjusts any constraints for supplying power between the grid and H-MG in secondary control.

The value of the total generated power (TGP) is shown in Figure. 12. As before PVS unit entering service begins (coming into operation), the power shortage is mainly supplied by purchasing power from the grid. As soon as PVS comes into operation, a major part of power shortage is supplied by MTS. During the period in which the power generated by PVS is increasing, the proposed algorithm tries to store excess power in BES, so that remainder of the time-wise SOC condition needs for secondary control can be fulfilled. After the occurrence of Scenario $\sharp 2$ and $\sharp 3$, MTS comes into operation with maximum capacity and the rest of the required power is supplied by the grid, such that, while maintaining the power balance constraints in the grid, the power exchange between the grid and H-MG, are also regulated.

\subsection{The Convergence Specifications/Comparison for EMS- $M A B C$ and EMS-MINLP Algorithms}

The proposed algorithm has been implemented for solving the energy management system- EMS problem in the C programming environment and has been tested by using a computer with the specifications CPU 2.6 GHz and RAM 4GB. For comparison, the execution time of the proposed algorithm and the absolute value of CPU time have been mentioned in 
Table 2: Average calculation time for the system under study

\begin{tabular}{lll}
\hline \hline & MINLP & MABC \\
\hline Execution time (s) & 8.23 & 1.14 \\
\hline
\end{tabular}

Table 2 for both algorithms. The obtained results show that in addition CPU can allocate less time for executing EMSMABC algorithm compared with EMS-MINLP, the following cases from the viewpoint of practical implementation must be considered:

- For the real-time implementation of EMS system in the MG the execution time of the algorithm has significant importance. Real-time in different papers have been reported between 3 minute to 5 minutes. Also, its reason is that this time is less than the adjustment time for protective relay reactions such as under-/over-voltage relay and etc. In addition, atmospheric variations usually needs more than 5 seconds for affecting PV systems and wind turbines. The system under study has a connection network by using IEC 61850 standard between central computer and emulators which have been used for simulating the performance of distributed generation sources. The required time for sending data (the optimum power of all the DER sources and the amount of RLD from the central computer to the simulators) is between 1.5 and 3 seconds. Furthermore, the necessary time for exchanging data among intelligent sockets present inside the emulators which have been connected to each other by using connection link under CAN protocol and operate as local controller is also less than 3 seconds. So, the sum of the required time for executing the algorithm and the required time for sending data and information exchange has become much less than 3 minutes in both of the algorithms.

- Other point which can be stated is that the problems related to unit commitment in the MGs are mainly largescale with a large number of variables; non-convex, nonlinear and belonging to non-deterministic polynomial time hard (NP-hard). With the increase of the number of variables, the algorithm execution time will increase in exponential progression. So, using heuristic algorithms which give close to optimal point can be considered as a solution for such problems. Finally, the proposed algorithm is not only just for the system under study with a number of low variables but in fact the main purpose of presenting it, is for solving networks with greater scale.

- Another point is about the convergence specification of the implemented algorithm. The proposed algorithm convergence specification for a time interval has been shown in Figure. 13. Maximum iteration is set to 100. As it is observed in the Figure, the algorithm has become convergent after 68 iterations. For showing the quality
Table 3: Comparison of solution quality for the system under study

$\begin{array}{llll}\text { Standard deviation } & \text { Average cost } & \text { Minimum cost } & \text { Algorithm name } \\ 0.0066 & 0.2279 & 0.22 & \text { EMS-MABC } \\ 0.0425 & 0.2745 & 0.23 & \text { EMS-MINLP }\end{array}$

of the presented solution by the EMS-MABC algorithm for the system under study, the efficiency of the algorithm for 100 iterations has been presented in Table. 3 with the results obtained from the realistic algorithm (MINLP) (presented in [14]) under cases such as average cost, minimum cost and standard deviation. As it is observed in Figure. 13, it is obvious that the EMS-MABC algorithm has reached its minimum cost after 68 iterations. While the EMS-MINLP algorithm has reached its minimum value after 83 iterations. As it is observed in Table. 3, the average value in the EMS-MABC algorithm is very close to the value of minimum cost and standard deviation is also much less than the other algorithm.

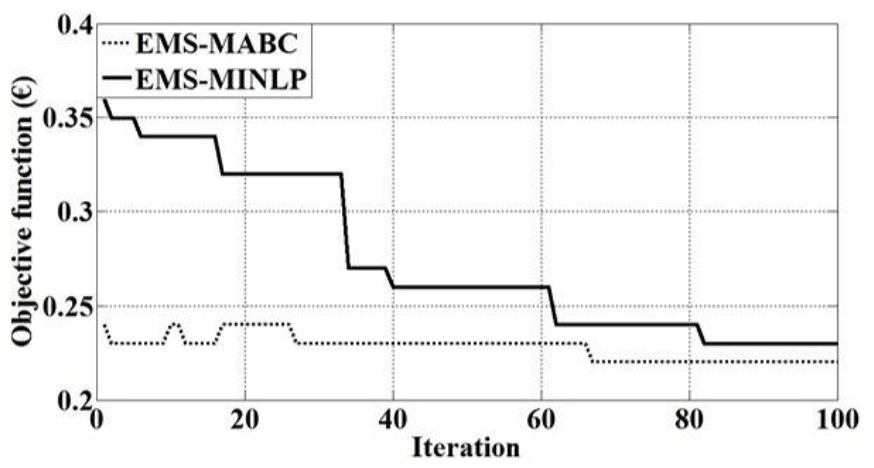

Figure 13: The characteristic of the convergence of the proposed algorithm for the system under study during a time interval

The H-MG operated by using the EMS-MABC algorithm has been able to perform optimum, robust, safe and stable function based on the following features:

1. A higher overall system input stream by taking into consideration the DG bids that accounts for the total consumed power by loads, as well, the total generated power by renewable sources;

2. A maximum satisfaction load rate by minimizing the operation cost in islanded mode;

3. An intelligent management of renewable units for longterm period intermittent durations and real-time forecasting by using real time schedule layer;

4. A precise adjustment of the set-point related to production units in real time schedule layer for the optimization of power distribution;

5. A fast precision-based algorithm reaction time and decision making by considering real-time forecast data for unwanted incidents resulting from the exit/entrance and/or increased/reduced capacity of non-dispatchable sources, as well, an increased demand for NRL. 


\section{Conclusions}

An intelligent EMS architecture and experimental evaluation has been successfully demonstrated for optimizing the power generation balance under DER electrical systems. The EMS-MABC energy management algorithm demonstrates the time-wise scheduling practical capability for a range of integrated power devices and demand loads. A significant reduction, being one of the primary feature, in the total electricity cost can be obtained. The priority of entering/exiting service of power generation sources and feeding responsive loads, based on the emulated objective function, demonstrates the inclusiveness of all the known constraints in DER. Comparison of experimental and simulation results shows the capability of the proposed algorithm to tolerate adequately the encounter with unwanted transients/incidents for the whole cycle of power generation to distribution operation.

The results highlights the effectiveness of the proposed EMS-MINLP algorithm under very high convergence time, an incentive for the smart grids energy management and distribution for H-MG applications in real-time. The demonstrated our H-MG topology seems a logical choice for achieving these goals, and our adaptive and recursive EMS-MABC algorithm devises our initiatives to show for optimum energy management that can certainly be envisioned for future hybrid multifunctional smart grid supply chain energy systems with a revolutionary architectures. The real-time energy management capability of a H-MG is shown to inter-operate at both; a connected to the grid and an independent from the grid, where the optimization algorithm based on ABC-method using a double layer control scheme, including a dedicated primary and secondary control. The optimum controllable set-points related to each of the variables can be obtained in hourly and real-time intervals by control schemes as follows:

- Adequate exploitation of non-dispatchable resources with the aim of increasing utilization with maximum capacity as a result of significant reduction of environmental population and also reduction of consumed electricity;

- Maximizing the energy stored in the BES and maintaining SOC at acceptable level for increasing BES system reliability and life cycle.

- Exchanging adequate power with the grid and efficient demand side management by applying DR and stable load feed with a reduced operational cost.

- The demonstrated results show the effectiveness of the proposed EMS-MINLP algorithm under very high convergence time, overall that can offer an incentive of smart real-time energy management, planning and distribution in power systems for H-MG applications.

\section{Acknowledgments}

This research was supported by institut de recerca en energia de catalunya (IREC), Barcelona, Spain. The authors are indebted to all of subjects who participated in the present project especially Dr. Andreas Sumper and Mrs.Fatemeh Azarinejadian.

This research is funded by the European Commission under contract "FP7-2013-NMP-ENV-EeB" through the Design4Energy project (Grant agreement no: 609380).

\section{References}

[1] K. Balasubramaniam, P. Saraf, R. Hadidi, E. B. Makram, Energy management system for enhanced resiliency of microgrids during islanded operation, Electric Power Systems Research, 137 (2016) 133-41.

[2] W. Alharbi, K. Raahemifar, Probabilistic coordination of microgrid energy resources operation considering uncertainties, Electric Power Systems Research, 128 (2015) 1-10.

[3] M. Marzband, N. Parhizi, M. Savaghebi, J. Guerrero, Distributed smart decision-making for a multimicrogrid system based on a hierarchical interactive architecture, IEEE Transactions on Energy Conversion, 31 (2) (2016) 637-48.

[4] A. Kargarian, M. Rahmani, Multi-microgrid energy systems operation incorporating distribution-interline power flow controller, Electric Power Systems Research, 129 (2015) 208-16.

[5] D. Tenfen, E. C. Finardi, A mixed integer linear programming model for the energy management problem of microgrids, Electric Power Systems Research, 122 (2015) 19-28.

[6] P. García, J. P. Torreglosa, L. M. Fernández, F. Jurado, R. Langella, A. Testa, Energy management system based on techno-economic optimization for microgrids, Electric Power Systems Research, 131 (2016) 49-59.

[7] T. Lv, Q. Ai, Y. Zhao, A bi-level multi-objective optimal operation of grid-connected microgrids, Electric Power Systems Research, 131 (2016) 60-70.

[8] M. Marzband, E. Yousefnejad, A. Sumper, J. L. Domínguez-García, Real time experimental implementation of optimum energy management system in standalone microgrid by using multi-layer ant colony optimization, International Journal of Electrical Power \& Energy Systems 75 (2016) 265-74.

[9] M. Marzband, M. Javadi, J. L. Domínguez-García, M. M. Moghaddam, Non-cooperative game theory based energy management systems for energy district in the retail market considering DER uncertainties, IET Generation, Transmission \& Distribution, 10 (2016) 2999-3009.

[10] P. Pinceti, M. Vanti, C. Brocca, M. Carnesecchi, G. Macera, Design criteria for a power management system for microgrids with renewable sources, Electric Power Systems Research, 122 (2015) 168-79.

[11] M. Marzband, M. M. Moghaddam, M. F. Akorede, G. Khomeyrani, Adaptive load shedding scheme for frequency stability enhancement in microgrids, Electric Power Systems Research, (2016) 1-11.

[12] M. Marzband, F. Azarinejadian, M. Savaghebi, J. M. Guerrero, An optimal energy management system for islanded microgrids based on multiperiod artificial bee colony combined with markov chain, IEEE systems journal, PP (99) (2015) 1-11.

[13] M. Marzband, M. Ghadimi, A. Sumper, J. L. Domínguez-García, Experimental validation of a real-time energy management system using multi-period gravitational search algorithm for microgrids in islanded mode, Applied Energy, 128 (0) (2014) 164-74.

[14] M. Marzband, A. Sumper, J. L. Domínguez-García, R. Gumara-Ferret, Experimental validation of a real time energy management system for microgrids in islanded mode using a local day-ahead electricity market and MINLP, Energy Conversion and Management 76 (0) (2013) 314 22.

[15] M. Marzband, N. Parhizi, J. Adabi, Optimal energy management for stand-alone microgrids based on multi-period imperialist competition algorithm considering uncertainties: experimental validation, International Transactions on Electrical Energy Systems, 30 (1) (2015) 12231.

[16] T. Logenthiran, D. Srinivasan, A. M. Khambadkone, Multi-agent system for energy resource scheduling of integrated microgrids in a distributed system, Electric Power Systems Research, 81 (1) (2011) 13848. 
[17] M. Marzband, A. Sumper, A. Ruiz-Álvarez, J. L. Domínguez-García, B. Tomoiagă, Experimental evaluation of a real time energy management system for stand-alone microgrids in day-ahead markets, Applied Energy 106 (0) (2013) 365-76.

[18] M. Marzband, A. Sumper, Implementation of an optimal energy management within islanded microgrid, International Conference on Renewable Energies and Power Quality (ICREPQ), Cordoba, Spain, 2014.

[19] M. Marzband, A. Sumper, M. Chindriş, B. Tomoiagă, Energy management system of hybrid microgrid with energy storage, The International Word Energy System Conference (WESC), Suceava, Romania, 2012.

[20] M. Marzband, A. Sumper, O. Gomis-Bellmunt, P. Pezzini, M. Chindris, Frequency control of isolated wind and diesel hybrid microgrid power system by using fuzzy logic controllers and PID controllers, in: Electrical Power Quality and Utilisation (EPQU), 2011 11th International Conference on, 2011, pp. 1-6.

[21] M. Moafi, M. Marzband, M. Savaghebi, J. M. Guerrero, Energy management system based on fuzzy fractional order PID controller for transient stability improvement in microgrids with energy storage, International Transactions on Electrical Energy Systems (2016) 1-20.

[22] M. Marzband, Experimental validation of optimal real-time energy management system for microgrids, Phd thesis, Departament d'Enginyeria Elèctrica, EU d'Enginyeria Tècnica Industrial de Barcelona, Universitat Politècnica de Catalunya (2013). 